\title{
Possible Effects of Drift Wave Turbulence on Magnetic Structure and Plasma Transport in Tokamaks
}

J. D. Callen 


\section{DISCLAIMER}

This report was prepared as an account of work sponsored by an agency of the United States Government. Neither the United States Government nor any agency Thereof, nor any of their employees, makes any warranty, express or implied, or assumes any legal liability or responsibility for the accuracy, completeness, or usefulness of any information, apparatus, product, or process disclosed, or represents that its use would not infringe privately owned rights. Reference herein to any specific commercial product, process, or service by trade name, trademark, manufacturer, or otherwise does not necessarily constitute or imply its endorsement, recommendation, or favoring by the United States Government or any agency thereof. The views and opinions of authors expressed herein do not necessarily state or reflect those of the United States Government or any agency thereof. 


\section{DISCLAIMER}

Portions of this document may be illegible in electronic image products. Images are produced from the best available original document. 


\section{Printed in the United States of America. Available from National Technical Information Service \\ U.S. Department of Commerce \\ 5285 Port Royal Road, Springfield, Virginia 22161 \\ Price: Printed Copy $\$ 4.00 ;$ Microfiche $\$ 3.00$}

This report was prepared as an account of work sponsored by the United States Government. Neither the United States nor the Energy Research and Development Administration/United States Nuclear Regulatory Commission, nor any of their employees, nor any of their contractors, subcontractors, or their employees, makes any warranty, express or implied, or assumes any legal liability or responsibility for the accuracy, completeness or usetulness of any intormation, apparatus, proauct or process disclosed, or represents that its use would not infringe privately owned rights. 
Contract No. W-7405-eng-26

FUSION ENERGY DIVISION

POSSIBLE EFFECTS OF DRIFT WAVE TURBULENCE

ON MAGNETIC STRUCTURE AND PLASMA

TṘANSPORT IN TOKAMAKS

J. D. Callen

Date Published: July 1977

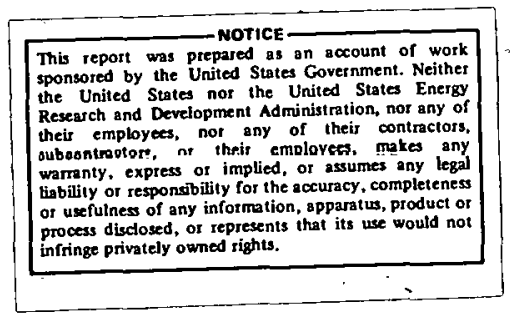

NOTICE This document contains information of a preliminary nature. It is subject to revision or correction and therefore does not represent a final report.

Prepared by the OAK RIDGE NATIONAL LABORATORY

Oak Ridgc, Tennessee 37830

operated by

UNION CARBIDE CORPORATION

for the

ENERGY RESEARCH AND DEVELOPMENT ADMINISTRATION

PSTRIBUTION OF THIS DOCUMENT IS ÚNLIMITED fly 


\section{ABSTRACT}

A new mechanism is proposed by which low leve1, drift wave type fluctuations, such as those observed in the ATC and TFR experiments $\left(\mathrm{e} \tilde{\phi} / \mathrm{T}_{\mathrm{e}} \leqslant 10^{-2}\right)$, can cause anomalous radial electron heat transport in tokamaks. The model is based on the fact that since transport processes parallel to the magnetic field are many orders of magnitude morc rapid than perpendicular ones, very small helically resonant magnetic perturbations (e.g., $\tilde{B}_{r} / B>\sqrt{X_{1} / X_{\|}} \sim 10^{-4}$ ). that cause field lines to move radially allow the parallel transport process to contribute to radial electron heat transport. We hypothesize that the small magnetic perturbations $\left(\tilde{B}_{\mathrm{r}} / \mathrm{B} \leqslant 10^{-3}\right)$ accompanying drift waves at any nonzero plasma $\beta$ (ratio of plasma pressure to magnetic energy density) are large enough to producc significant effects in present tokamak experiments. The helical magnetic component of drift waves produces magnetic island structures whose spatial widths $(\delta \sim 0.5 \mathrm{~cm})$ can easily exceed the ion gyroradius. In a drift wave oscillation period, electrons circumnavigate a magnetic island, whereas the slower moving ions see only a tilt of the magnetic field lines. Thus, electrons try to diffuse radially more rapidly than ions; however, a radial potential builds up on a very short time scale to confine the electrons electrostatically and thereby keep the particle diffusion ambipolar. Nonetheless, this parallel electron diffusion process does cause net radial electron heat conduction through an ensemble of closely packed island structures. The heat conduction coefficient is estimated to be $x_{e}^{\delta} \sim(3 / 16)(\nu+\gamma) \delta^{2}$, where $\nu$ is the electron-ion collision frequency and $\gamma$ is the drift wave growth rate, or inverse island correlation time. Other effects that these magnetic flutters may have on plasma transport and runaway electron processes are also discussed. 


\section{INTRODUCTION}

Smal1, resonant magnetic perturbations can cause significant distortions of the magnetic surfaces ${ }^{1}$ in tokamaks. Magnetic perturbations can be caused by inhomogeneities in the external coils producing the confining magnetic field or by the currents induced by collective oscillations in the confined plasma. The magnetic islands induced by irregularities in the external coil system are typically narrow in width, and well separated in radius, ${ }^{2}$ or at least can be designed to be so. Thus, even when they occur they are expected to have little effect on overall plasma transport. Similarly, in reasonably quiescent discharges, low mode number kink and tearing instabilities produce substantial distortions of the magnetic topology ${ }^{3}$ and plasma transport ${ }^{4}$ only in the near vicinity of low order rational surfaces. While internal disruptions, which are apparently due to a repetitive cycle of ohmic heating with consequent $m=1$ tearing mode growth through a nonlinear flattening stage, $b$ affect the entire central core region where the rotational transform exceeds $2 \pi$, they do not seein tu have much direct influence on gross plasma confinement properties. The major disruptions, which can be avoided by keeping the rotational transform small enough, apparently result ${ }^{6}$ from a coupling of two or more low mode number instabilities and seem to be due to a complete rearrangement of the magnetic topology in the plasma. Various ergodicity and magnetic braiding arguments ${ }^{7}$ are often invoked to provide a mechanism for anomalous transport between low order rational surfaces; however, it seems ${ }^{8}$ that in the absence of major disruptions, low mode number macroinstabilities do not have a significant effect on the overall plasma confinement in a tokamak. The hypothesis advanced in this paper is that the high mode number 
magnetic perturbations induced by the microscopic drift wave type turbulence that has been observed experimentally, ${ }^{9}$ and which is probably not purely electrostatic, ${ }^{10}$ can induce densely packed magnetic island structures and thereby strongly affect radial plasma transport in tokamaks. A similar model of effects of stochastic magnetic turbulence in a fluid model was proposed and found to be important in determining the electron energy confinement in the Zeta device. ${ }^{11}$ Also, Rechester ${ }^{12}$ has advanced a model of radial electron heat transport due to stochastic magnetic field lines in a collisional plasma.

This paper is organized as follows. In Sect. 2 we briefly discuss the physical mechanism through which small magnetic perturbations can affect radial transport processes. Then, in Sect. 3 we estimate the magnitude and spatial structure of the magnetic perturbations due to drift waves. The magnetic island structures induced by the drift wave magnetics are briefly discussed in Sect. 4. Next; in Sect. 5 the effects that these magnetic island structures have on particle and energy transport in a tokamak are derived in some detail. Our conclusions are summarized in Sect. 6 .

2. PHYSICAL MECHANISM FOR MAGNETIC PERTURBATION EFFECTS ON TRANSPORT The basic physical effect that allows very small magnetic perturbations to affect plasma transport so readily is that plasma transport is much faster along magnetic field lines than perpendicular to them. Thus, magnetic perturbations that cause field lines to flutter radially in a tokamak plasma can allow a portion of the very rapid, parallel transport processes to contribute to radial plasma transport. If the collisional mean free path $(\lambda)$ in a collisional, magnetized plasma is so small that 
particles follow the radial motion of the magnetic field lines caused by the magnetic perturbations but do not go far enough along a field line to complete a significant fraction of a magnetic island, the effective radial heat transport is apparent $1 y^{4,12}$

$$
x_{\text {eff }} \sim x_{1}+\left(\tilde{B}_{r} / B\right)^{2} x_{\|}
$$

where $\chi_{1}$ and $\chi_{\|}$are the heat transport coefficients perpendicular and parallel to the magnetic field, ${ }_{B}{ }_{r}$ is the (stochastic ${ }^{12}$ ) radial component of the magnetic perturbation, and $B$ is the strength of the confining magnetic field. In a collisional plasma $x_{\|} / x_{1} \sim \lambda^{2} / \rho^{2}$, where $\rho$ is the particle gyroradius. Thus, magnetic perturbations may have a significant effect on radial heat transport processes in a collisional plasma if

$$
\tilde{\mathrm{B}}_{\mathrm{r}} / \mathrm{B}>\sqrt{\chi_{1} / X_{\|}} \sim \rho / \lambda
$$

the ratio of $\rho$ to $\lambda$ can easily be as small as $10^{-6}$ for electrons and $10^{-1}$ for ions. 'lhis is a miniscule level of magnetic fluctuations.

\section{MAGNETIC PERTURBATIONS INDUCED BY DRIFT WAVES}

While the magnetic perturbations produced by drift waves in a tokamak are small, they can easily be significant in the sense of Eqs. (1) and (2). Drift waves are usually analyzed in the electrostatic limit, which Is valid in the limit of vanizhingly smull $\beta, \dot{i} . e ., \beta \ll m_{e} / m_{i}$, where $\beta$ is the ratio of plasma pressure to magnetic energy density. However, for tokamak plasmas of present interest in which $\beta \sim 10^{-4}:-10^{-1}$, the nonelectrostatic effects can be significant. ${ }^{10}$ The magnetic perturbation component of drift waves is caused by the fluctuating electric current produced by the difference between the wave-induced perpendicular drifts 
$\left(V_{1}\right)$ of the ions and electrons. ${ }^{13}$ Since for these low frequency

oscillations the perturbed current must be divergence-free, the component of the perturbed current parallel to the magnetic field is given by $\tilde{J}_{\|}=\left(i / k_{\|}\right) \underline{\nabla} \cdot n e\left(V_{-1}-V_{1}\right) \cdot$ Taking into account the polarization and finite ion gyroradius drifts, one obtains ${ }^{13}$ $\tilde{J}_{\|} \simeq-\omega\left(\mathrm{nm}_{i} c^{2} / \mathrm{B}^{2}\right)\left(k_{1}^{2} / \mathrm{k}_{\|}\right)\left[1+\left(\mathrm{T}_{\mathrm{i}} / \mathrm{T}_{\mathrm{e}}\right)\left(\omega_{*} \mathrm{e} / \omega\right)\right] \tilde{\phi}$. Here $\tilde{\phi}$ is the perturbed potential, $\omega_{\star_{e}} \equiv \mathrm{k}_{\theta}\left(\mathrm{cT}_{\mathrm{e}} / \mathrm{eB}\right)(\mathrm{d} \ln \mathrm{n} / \mathrm{dr})$ is the electron diamagnetic drift velocity, and in the sheared magnetic field characteristic of a tokamak $k_{\| l}(x)=k_{\theta} x / L_{s}$, where $x \equiv r-r_{0}$ is the radial distance away from a rational surface and $\mathrm{L}_{\mathrm{s}}^{-1}=(\mathrm{r} / \mathrm{Rq})\left(\mathrm{q}^{-1} \mathrm{dq} / \mathrm{dr}\right)$ is the characteristic shear length of the magnetic field. The perturbed current induces, through Ampere's law, a perturbed magnetic field $\tilde{\mathrm{B}}_{-1}$ that is perpendicular to the equilibrium magnetic field $\underline{B}$ but helically aligned with it at the rational surface:

$$
\tilde{B}_{1} / B \simeq i\left(\omega / k_{\|}(x) V_{A}\right)\left(k_{\perp} \hat{\rho}_{i}\right)\left(V_{s} / V_{A}\right)\left(1+T_{i} \omega_{*} e^{/ T} e^{\omega)\left(e \tilde{\phi} / T_{e}\right.}\right),
$$

where $V_{A} \equiv B / \sqrt{4 \pi m_{i}}$ is the Alfvén speed, $V_{S} \equiv \sqrt{T_{e} / m_{i}}$ is the ion sound speed, and $\hat{\rho}_{i}=V_{s} / \Omega_{i}$ is the ion gyroradius measured at the electron temperature. Note that: (1) $\tilde{B}_{\perp}$ bends or twists the magnetic field lines but does not compress them; (2) since $v_{s} / V_{A}=\sqrt{\beta_{e} / 2}$, the strength of the magnetic perturbations is proportional to $\sqrt{\beta_{e}}$; and (3) this expression for $\tilde{B}_{1}$ is valid only for $k_{\theta}>\partial / \partial x$ and $\omega<k_{\|}(x) V_{A}$, i.e., $x>x_{\Lambda}$ where $x_{A} \equiv \omega L_{s} / k_{\theta} V_{A} \simeq \hat{\rho}_{i}\left(L_{s} / r_{n}\right)\left(V_{s} / V_{A}\right)$. The equation governing the full radial or $x$ dependence of $\tilde{B}_{r}$ is derived and discussed in Ref. 14 . For typical parameters in present tokamak experiments $\left(\mathrm{k}_{1} \hat{\rho}_{\mathrm{i}} \leqslant 1, \mathrm{~V}_{\mathrm{s}} / \mathrm{V}_{\mathrm{A}}\right.$ $\left.\leqslant 1 / 20, \mathrm{~T}_{i} \leqslant \mathrm{~T}_{\mathrm{e}}, \mathrm{e} \tilde{\phi} / \mathrm{T}_{\mathrm{e}} \leqslant 10^{-2}\right)$, we have $\tilde{\mathrm{B}}_{\perp} / \mathrm{B} \leqslant 10^{-3}\left(\omega / \mathrm{k}_{\|} \mathrm{V}_{\mathrm{A}}\right) \leqslant 10^{-3}$; 
comparison of this estimate with Eq. (2) indicates that these magnetic perturbations could indeed have significant effects on radial transport processes in tokamaks.

4. DRIFT WAVE MAGNETICS: EFFECTS ON MAGNETIC TOPOLOGY

These magnetic perturbations can change the magnetic topology of a tokamak by forming thin, high order magnetic islands ${ }^{15}$ at surfaces where the pitch of the field lines and the pitch of the perturbations are the same. The radial variations of the perturbed potential $\tilde{\phi}$ and the mägnertc field are shown in Figs. $1(a)$ and $1(b)$. In Fig. 1(c) we illustrale the magnetic islands formed by adding this magnetic perturbation to the helical component of the equilibrium magnetic field near a rational surface, ${ }^{16}$ $B_{n} \simeq-\left[B_{\theta}-(r / R)(n / m) B_{\phi}\right] \simeq x B_{\theta} q^{-1} d q / d r$ where $n \equiv n \phi-m \theta$ is the helical angle variable for a given perturbation localized about the rational surface at which $q\left(r_{S}\right)=\dot{m} / n$; here, $\phi$ and $\theta$ are respectively the toroidal and poloidal angle coordinates and $n$ and $m$ are respectively the toroidal and poloidal mode numbers. It can be shown that for the magnetic perturba-

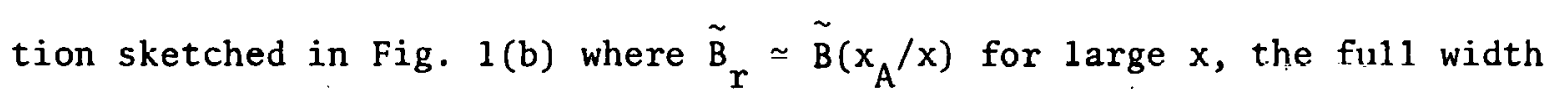
of the induced magnetic island structure is given by 15

$$
\delta \simeq 2\left[\left(\tilde{\mathrm{B}} / \tilde{\mathrm{B}}_{\theta}\right)\left(2 \mathrm{x}_{\mathrm{A}} \mathrm{r}_{\mathrm{s}} / \mathrm{nq}^{\wedge}\right)\right]^{1 / 3}
$$

For typical parameters in present tokamaks $\left[\tilde{B} / B_{\theta} \sim\left(B / B_{\theta}\right)(\tilde{B} / B) \sim 1 / 30\right.$, $\mathrm{x}_{\mathrm{A}} \sim 0.1 \mathrm{~cm}, \mathrm{r}_{\mathrm{s}} \sim 10 \mathrm{~cm}, \mathrm{q}^{-} \sim 0.1 \mathrm{~cm}^{-1}$, and $\left.\mathrm{n} \sim 45\right]$, we obtain $\delta \sim 0.5 \mathrm{~cm}$. The distance a magnetic field line travels in tracing out a particular magnetic island is given by $2 \pi R N$, where ${ }^{i \overline{5}}$

$$
N \sim q / n \delta q^{\prime}
$$


is the winding number, i.e., the number of times a field line circumnavigates the torus in one complete circumnavigation of an island. For typical parameters $\left(\mathrm{n} \sim 45, \delta \sim 0.5 \mathrm{~cm}, \mathrm{q}^{-} \sim 0.1 \mathrm{~cm}^{-1}\right.$, $q \sim 2$ ), we obtain $N>1$. If the magnetic perturbation were nearly uniform in space, such as occurs in tearing modes or externally induced perturbations, then ${ }^{15}$ in Eq. (4) the $1 / 3$ power becomes $1 / 2$, the $x_{A}$ is replaced by 2, and the $\mathrm{N}$.in Eq. (5) becomes a factor of 4 larger.

\section{DRIFT WAVE MAGNETICS: EFFECTS ON PLASMA TRANSPORT PROCESSES}

The effects that drift wave magnetics have on electrons are quite different from the effects on ions. In one drift wave period, since $\omega \gg v_{i} / 2 \pi R N$, ions do not move far enough along the magnetic field lines to be aware of the magnetic island structure. Rather, they see only a small stochastic variation in the direction of the magnetic field lines. The perturbed ion distribution function response due to the magnetic perturbations can thus be calculated from the linearized Vlasov equation in the usual way. The quasilinear effect of this perturbation can be shown to be smaller by a factor $\beta$ [because of the $V_{s} / V_{A} \sim \sqrt{B}$ factor in Eq. (3)] than the usual $\underline{\tilde{E}} \times \underline{B}$ electrostatic quasilinear effect of the drift waves on ion transport processes; hence it is negligible.

In contrast to the ions, electrons are aware of the full magnetic island structure since they circumnavigate the islands many times in a wave or growth period, i.e., $\omega \ll v_{e} / 2 \pi R N$. To compute the electron distribution function in the presence of the islands, we begin with the lowest order drift-kinetic equation

$$
\frac{\partial f}{\partial \dot{t}}+\left(v_{\|} \underline{n}+v_{E}\right) \cdot \frac{\partial f}{\partial \underline{x}}+q v_{i \|}\left(-\frac{1}{c} \frac{\partial A_{\|}}{\partial t}\right) \frac{\partial f}{\partial E}=C(f),
$$


where we have neglected some finite Larmor radius effects because they have no significant effect on the analysis that follows. Magnetic perturbations come into Eq. (6) both directly through the $\partial A_{\|} / \partial t$ term and indirectly through the unit vector along $\underline{B}$, which becomes

$$
\underline{\mathrm{n}} \equiv \underline{\mathrm{B}} / \mathrm{B} \simeq \underline{\mathrm{n}}_{\mathrm{o}}+\underline{\tilde{\mathrm{B}}}_{-1} / \mathrm{B}
$$

in the presence of a magnetic perturbation $\tilde{B}_{1}$. Here $n_{-n}=\left(B_{\phi} \hat{\phi}+\hat{B}_{\theta} \hat{\theta}\right) / B$ is the unit vector along the equilibrium magnetic field. Taking account of these magnetic perturbation effects, neglecting the curvalure drift term since it will not contribute to the ultimate answers, linearizing Eq. (6) about a local Maxwellian distribution $f f=f_{m}+\tilde{f}$, with $f_{m}=n_{e}(2 \pi T / m)^{-3 / 2}$ $\exp [-(E-q \Phi) / T]$, and $\left.E=m v^{2} / 2+q \Phi\right\}$, and assuming the lowest order $\tilde{f}$ is an adiabatic response $\left(\tilde{f} \equiv-q \tilde{\phi} f_{m} / T+g\right)$, we obtain for the equation governing the nonadiabatic electron response 10

$$
\left(\lambda / \partial t+v_{\|} n_{-0} \cdot \underline{\nabla}-C\right) g=-i\left(\omega-\omega_{*}^{T}\right) q \ddot{\phi}_{f_{m}} / T-v_{\|}\left(\tilde{B}_{r} / B\right)\left(1-\omega / \omega_{*}^{T}\right) \partial f_{m} / \partial r .
$$

Here, we have assumed that $\tilde{\mathrm{f}}, \tilde{\phi}, \tilde{\tilde{B}}_{\mathrm{r}} \sim \exp [i(n \phi-m \theta-\omega t)]$, used the fact that $\tilde{B}_{r} \simeq i k_{\theta} A_{\|}$, and defined in the $\underline{E} \times \underline{B}$ rest frame where $\Phi=0$, $\omega_{*}^{T} \equiv-k_{\theta}\left(c^{\prime} l^{\prime} / q B\right) \partial \psi_{n} f_{m} / \partial r=\omega_{*}\left[1+\eta_{e}(E / T-3 / 2)\right]$, with $\omega_{*}=\left(c k_{\theta} T / c B\right)$ $\partial \ln \mathrm{n} / \partial \mathrm{r}$, and $\mathrm{n}_{\mathrm{e}} \equiv 2 \ln \mathrm{T} / \partial \ln _{\mathrm{n}} \mathrm{n}_{\mathrm{e}}$. Integrating Eq. (8) over the characteristics of the left side of the equation, we obtain the usual perturbed electron response, which is responsible for the usual quasilinear particle and energy transport fluxes in the plasma. The $\ddot{\mathrm{B}}_{\mathrm{r}}^{2}$ contribution to $\mathrm{Eq}$. (1) is obtained by assuming that the collision operator in Eq. (8) gives the dominant contribution to the left side of Eq. (8), (i.e., $\left.C \sim v \gg, \omega, w_{b}\right)$ and then computing the contribution to $g$ and the heat flux due to the inhomogeneous $\tilde{B}_{r}$ term on the right of Eq. (8). 
Here, we wish to determine the additional contribution $\left(g_{\delta}\right)$ to the perturbed electron distribution function due to the presence of the magnetic islands. This contribution is obtained by calculating the evolution of an initially Maxwellian distribution as it propagates along the magnetic island structure. Since the definition of a magnetic field line is $d \ell / B=d x / \tilde{B}_{r}$, we can identify $v_{\|} \tilde{B}_{r} / B=d x / d t$ as the radial velocity of a particle moving along a magnetic field line within the magnetic island structure. Thus, we see from Eq. (8) that the island contribution to the perturbed electron distribution function is governed by the equation

$$
\left(\partial / \partial t+v_{\|} n_{-o} \cdot \underline{\nabla}+\nu\right) g_{\delta}=-(d x / d t)\left(1-\omega / \omega_{*}^{T}\right) \partial f_{m} / \partial r,
$$

where for simplicity we have taken the collision operator to be of the Krook type with an energy-independent collision frequency $v$. Integrating over the characteristics of the left side of Eq. (9), we readily obtain for the time-asymptotic response ( $\operatorname{Im} \omega>0$ )

$$
g_{\delta} \simeq-\sum_{\omega} e^{-i \omega t}\left(1-\omega / \omega_{*}^{T}\right)\left(\partial f_{m} / \partial r\right) \int_{-\infty}^{t} d t^{\prime}(d x / d t)^{\prime} \exp \left[-i(\omega+i \nu)\left(t^{\prime}-t\right)\right]
$$

in which the primes denote integration along the electron trajectories, and $\tilde{B}_{r} \equiv \sum_{\omega} \hat{B}_{r} e^{-i \omega t}$ has been decomposed into its temporally and spatially varying parts such that $d x / d t=v_{\|} \hat{B}_{r} / B$.

To proceed further we must specify. a trajectory for the radial motion of electrons as they circumnavigate the magnetic islands. Given the complexity of the island structures [cf. Fig. l(c)], this is very difficult to do exactly. However, since the radial velocity $\mathrm{dx} / \mathrm{dt}$ must be periodic in t. with a period of $\tau_{\delta}=2 \pi R N / v_{\|}$, we can cxpand $d x / d t$ in a 
Fourier series in the island circumnavigation frequency: $d x / d t=\omega_{\delta}(\Delta / 4) \times$ $\sum_{\mathrm{p}} C_{\mathrm{p}} \exp \left(\mathrm{ip} \omega_{\delta} t\right)$, where $\omega_{\delta} \equiv 2 \pi / \tau_{\delta}=v_{\|} / \mathrm{RN}, \Delta / 4$ is the half-width of a given contour within a magnetic island. From the nature of electron motion within the islands, we observe that $C_{p}=0$ if $p$ is zero or an even integer. While this general form of $\mathrm{dx} / \mathrm{dt}$ can be used, for simplicity in what follows, we assume that $C_{1}=C_{-1}=1 / 2, C_{n \neq \pm 1}=0$ and then have for the electron trajectory simp1y

$$
d x / d t=\omega_{\delta}(\Delta / 4) \cos \omega_{\delta} t, x=x_{0}+(\Delta / 4) \sin \omega_{\delta} t
$$

Performing the time-history_integration in Eq. (10) along this triajectory, we obtain

$g_{\delta} \simeq-\sum_{\omega} e^{-i \omega t}\left(1-\omega / \omega_{*}^{T}\right)(\Delta / 4)\left(\partial f_{m} / \partial r\right)\left\{\sin \omega_{\delta} t-i\left[(\omega+i \nu) / \omega_{\delta}\right] \cos \omega_{\delta} t+\ldots\right\}$

in which we have made an expansion in $(\omega+i \nu) / \omega_{\delta} \ll 1$. It is readily seen from Eq. (11) that the sin $\omega_{\delta} t$ term in Eq. (12) represents a flattening of the total electron distribution function $\left(f_{m}+\tilde{f}\right)$ around

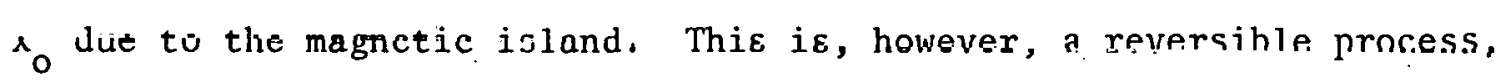
as evidenced by the $e^{-i \omega t}$ coefficient. The $\left[(\omega+i \nu) / \omega_{\delta}\right] \cos \omega_{\delta} t$ correction term to the flattening represents the irreversible processes of wave growth and collisional changes in the island-averaging period $\tau_{\delta}$ through diffusion in $v_{\|}$, which will ultimately lead us to obtain net transport in the radial direction from this perturbation.

The induced particle transport flux is calculated from the $\int d^{3} v$ moment of Eq. (6), which can be written as $\partial n / \partial t+(1 / r)(\partial / \partial r) \times$

$$
\begin{aligned}
& {\left[r\left(\Gamma_{r}^{\delta}+\Gamma_{o}\right)\right]=0 \text { where }} \\
& \quad \Gamma_{r}^{\delta}=\left\langle\int d^{3} v\left(v_{\|} \tilde{B}_{r} / B\right) g_{\delta}\right\rangle
\end{aligned}
$$


and $\Gamma_{0}$ is the sum of the usual neoclassical and quasilinear particle fluxes. Here, the average is an island and wave period average:

$$
\langle A\rangle \equiv(\pi N)^{-1} \int_{-\pi N / 2}^{\pi N / 2} \mathrm{~d} \phi(2 \pi)^{-1} \int_{-\pi}^{\pi} \mathrm{d} \theta(\omega / 4 \pi M) \int_{-M / \omega}^{M / \omega} \mathrm{d} A \text {. }
$$

Again splitting $\tilde{B}_{\mathrm{r}}$ into its spatially and temporally varying parts, and noting that $v_{\|} B_{r} / B=d x / d t$, we find.

$$
\Gamma_{\mathrm{r}}^{\delta} \simeq-\sum_{\omega \neq \omega_{\mathrm{mn}}} \frac{1}{2}(\Delta / 4)^{2} \int d^{3} v\left(1-\omega / \omega_{*}^{\mathrm{T}}\right)\left(\partial \mathrm{f}_{\mathrm{m}} / \partial \mathrm{r}\right)(\gamma+\nu)
$$

in which $\omega_{m n}$ are the set of rational surface drift frequencies over the radius of the plasma. Since the radial derivative of the Maxwellian distribution (at constant E) is given by $\partial f_{m} / \partial r=f_{m}[d \ell n n e / d r-(e / T)$ $\mathrm{d} \Phi / \mathrm{dr}+(E / \mathrm{T}-3 / 2) \mathrm{d} \ell \mathrm{T} / \mathrm{dr}]$, upon evaluating Eq. (14), we obtain

$$
\Gamma_{r}^{\delta} \simeq-\sum_{\omega=\omega_{m n}} \frac{1}{2}(\Delta / 4)^{2}(\nu+\gamma) n_{e}\left[d \ln n_{e} / d r-(e / T) d \Phi / d r-\omega e B /\left(c_{\theta} t_{e}\right)\right]
$$

Now, since the ion particle flux is the much smaller quasilinear one, the radial electron particle flux just derived would cause a nonambipolar radial flow of electrons. This causes a radial potential to build up on a very short time scale $\left(\ll \omega_{\delta}^{-1}\right)$ just so as to make the particle flux ambipolar. From Eq. (15), a sufficient condition for having this occur is that the sum of the terms within the brackets in Eq. (15) vanish, thereby prescribing an ambipolar potential of the form

$$
\left(e / T_{e}\right) d \Phi / d r=\left(1-\omega_{m n} / \omega_{*}\right) d \ln n_{e} / d r .
$$

Note that in the rest frame of the plasma the electrons are electrostatically confined in the radial direction. That is, they are no longer magnetically 
confined since they can flow radially along magnetic field lines; instead, the relatively immobile ions hold them back electrostatically. Nonetheless, the magnetic islands can produce a substantial enhancement of the radial electron heat flux.

Before calculating the radial electron heat flux, there is another effect we must take into account. We have calculated $g_{\delta}$ within only a single closed magnetic island of width $\Delta / 2<\delta / 2$ [cf. Fig. 1(c)]; recall that $\delta / 2$ is the full width of the island from one side of the sepurulris to the rational sirface. However, since the separatrices, which are isotherms, connect the island structures on opposite sides of the rational surface, on a very short time scale $\left(\leqslant \omega_{\delta}^{-1}\right)$ the electron temperature profile will be flattened over the entire magnetic island structure. Thus, $\Delta / 4$ in Eq. (12) should be replaced by $\delta / 2$. Taking this and the specification of the ambipolar potential [Eq. (16)] into account, Eq. (12) becomes

$$
\begin{aligned}
g_{\delta} \simeq-\sum_{\omega} c^{-i \omega t}\left(1-\omega / \omega_{\star}^{T}\right)(\delta / 2)(E / T-3 / 2)\left(f_{m} \operatorname{den} T / d r\right) x \\
\\
\left\{\sin \omega_{\delta} t-i\left[(1 \omega+i \nu) / \omega_{\delta}\right] \cos \omega_{\delta} t+\ldots\right\} .
\end{aligned}
$$

Then, calculating the island-induced electron heat flux in the same manner as the particle flux, except for the insertion of the energy moment factor $m v^{2} / 2$, we obtain

$$
Q_{r}^{\delta} \simeq-n_{e} x_{e}^{\delta} \mathrm{dT}_{\mathrm{e}} / \mathrm{dr}
$$

where

$$
x_{e}^{\delta} \simeq(3 / 4) \sum_{\omega=\omega_{m n}}(\nu+\gamma)(\delta / 2)^{2}
$$

Note that this is essentially the sum of the irreversible radial electron 
heat conduction within each island belonging to the ensemble of magnetic islands present in the plasma. The physical implication of this "weak turbulence" result is that electron heat diffuses radially with a step size of half the magnetic island width $(\delta / 2)$, at a characteristic rate given by. the sum of the drift wave growth rate and the $\left(90^{\circ}\right)$ collisional scattering rate for changing the island circumnavigation period $\tau_{\delta}$, which is proportional to $v_{\|}$. In a fully developed turbulent state such as might be derived from strong turbulence theory, ${ }^{17}$ the $\gamma$ would apparently be replaced by the "birth and death rate" for the various magnetic islands. In an abstract sense, Eq. (18) would be replaced by $x_{e}^{\delta} \sim \int d \tau(d x / d t)_{t}(d x / d t)_{t+\tau}$, where $d x / d t$ is the electron trajectory given by Eq. (11). This form illustrates the basic features of transport in the presence of the almost oscillatory radial motion. Namely, transport occurs only through the irreversible effects of collisions and island evolution, with net transport occuring only if the "death" phase of the islands is sufficiently different from their growth phase, i.e., the net radial heat transport is irreversible through an entire island life cycle. In order for there to be electron heat transport over the entire radius, the various magnetic islands represented by the $\omega_{\mathrm{mn}}$ in Eq. (18) must be closely packed or perhaps even overlap. Since the spatial separation of rational surfaces having the same $n$ is only $\Delta x \sim\left(\mathrm{nq}^{-}\right)^{-1} \sim 0.2 \mathrm{~cm}$ for typical parameters, such proximity is quite probable for the various $m$ modes having the same $\mathrm{n}$, and virtually certain for the doubly denumerable infinity of combinations of $m$ and $n$ admissible for the modes of interest. 
Note that, with the exception of the general form of the spatial structure shown in Fig. 1, we have used little or no specific information about the particular types of drift instabilities involved. Thus, the drift wave growth rate in Eq. (18) can be that due to one or all of the destabilizing effects of trapped electrons, finite ion Larmor radius, plasma current, etc. on drift waves. In addition, our treatment of the effects of drift wave magnetics would also apply. to the hypothesized high mode number drift-tearing modes, 18 with the island width $\delta$ and toroidal winding number $N$ modified as indicaled at the end of Sect. 4 for these $\tilde{B}_{r} \sim$ constant modes. Also, since the drift wave magnetics modify the magnetic topology so profoundly and stochastically, it seems that the highly ordered convection of wave energy away from a rational surface by a smoothly sheared magnetic field structure will. no longer occur. Rather, the complicated, overlapping magnetic island structures should reflect a significant portion of the outgoing wave energy and thereby greatly diminish if not totally defeat the usual magnetic shear 3tabilization mechanism 10,15 for drift wavẹ.

Since the electron heat transport due to the magnetic island formation scales as $x_{e}^{\delta} \sim \delta^{2} \sim\left(e \tilde{\phi} / T_{e}\right)^{2 / 3}$, whereas the typtcal quasilinear. estimate gives $X_{e}^{Q L} \sim\left(e \tilde{\phi} / T_{e}\right)^{2}$, at low fluctuation levels the magnetics effects are likely to dominate. While a condition on $e \ddot{\phi} / T_{e}$ for which $\chi_{e}^{\delta}>\chi_{e}^{Q L}$ can be written down, it is too complicated to be useful. As an illustration of the magnitudes involved, we note that for the fluctuations observed in $\operatorname{ATC},{ }^{9}$ we use the parameters $\nu_{e i} \sim 2 \times 10^{5} \mathrm{sec}^{-1}, \gamma \sim 2 \times 10^{5} \mathrm{sec}^{-1}$, $k \rho_{i} \leqslant 1, \gamma / \omega_{*} \sim 1 / 2, v_{s} \sim 3 \times 10^{7} \mathrm{~cm} / \mathrm{sec}, r_{n} \sim 10 \mathrm{~cm}, e \tilde{\phi} / \mathrm{T} \mathrm{e}^{\sim 5 \times 10^{-3}}$, and obtain $\delta \sim 0.5 \mathrm{~cm}$; hence, $\chi_{\mathrm{e}}^{\delta} \sim 2 \times 10^{4} \mathrm{~cm}^{2} / \mathrm{sec}$ vs $\chi_{\mathrm{e}}^{\mathrm{QL}} \sim 4 \times 10^{3} \mathrm{~cm}^{2} / \mathrm{sec}$. 
Similarly, for the TFR experiment ${ }^{9}$ we estimate that $\gamma \sim \nu_{e i} \sim 3 \times 10^{5}$, $\gamma / \omega_{*} \sim 1 / 2, v_{s} \sim 4 \times 10^{7} \mathrm{~cm} / \mathrm{sec}, r_{n} \sim 20 \mathrm{~cm}, e \tilde{\phi} / \mathrm{T}$ e $\sim 10^{-3}$, and obtain $\delta \sim 0.25 \mathrm{~cm}$; hence $\chi_{e}^{\delta} \sim 7.5 \times 10^{3} \mathrm{~cm}^{2} / \mathrm{sec}$ compared to $X_{e}^{Q L} \sim 4 \times 10^{2}$ $\mathrm{cm}^{2} / \mathrm{sec}$. In both these cases $\chi_{e}^{\delta} \gg \chi_{e}^{Q L}$ and the electron energy confinement time estimated from $\tau_{E e} \sim a^{2} / 4 \chi_{e}^{\delta}$ is close to that observed experimentally.

Finally, we note that the magnetic flutter induced by the drift wave turbulence may explain some previously incomprehensible characteristics of runaway electrons in tokamaks. Moderate energy runaway electrons, which are not significantly affected by the ambipolar potential and whose radial drift off of a flux surface $\left(x_{s}=q v_{e} / \Omega_{e}\right)$ is smaller than the island width $(\delta)$, should experience a radial diffusion rate of about $x_{e}^{\delta}$ in magnitude. This diffusion mechanism might explain the observations on ST, Pulsator, and LT-3 ${ }^{19}$ on runaway electron production, from which it was inferred that the confinement time for the moderate energy runaways was comparable to the energy containment time of the plasma. For very high energy runaways whose radial excursions pass over many magnetic islands ( $\left.x_{s} \gg \delta\right)$, we would expect the radial diffusion rate to be substantially reduced. This effect might explain the $D \sim 10^{2} \mathrm{~cm}^{2} / \mathrm{sec}$ radial diffusion of very high energy ( $\sim 5-10 \mathrm{MeV}$ ) runaway electrons observed ${ }^{20}$ in the ORMAK experiment. 


\section{CONCIIIISTINN}

We have shown that the small magnetic fluctuations induced by the primarily electrostatic drift wave turbulence in tokamaks can easily split the flux surface topology into closely packed, high order magnetic island structures. These islands cause a flattening of the electron distribution function near a rational surface. The imposition of an ambipolar diffusion constraint leads to the determination of an ambipolar radial electric field as given by Eq. (16). This radial electric field provides electrostatic containment of the electrons. Then, except for some possible effects on runaway electrons, the only residual effect of the magnetic flutter is to enhance the radial electron heat conduction up to a value given by Eq. (18), which appears to be in reasonable agreement with available experimental data. These drift wave magnetics effects apparently simply add to the usual neoclassical and $\tilde{E} \times \underline{B}$ quasilinear or strong turbulence transport processes. The important generic point of this study is the fact that very small magnetic perturbations indirectly induced by low frequency turbulence that is primarily electrostatic can have very substantial effects on the magnetic topology and plasma transport in magnetic confinement devices. 


\section{ACKNOWLEDGMENTS}

First and foremost, the author gratefully acknowledges the encouragement and many useful discussions of the concepts developed in this work with K. T. Tsang and B. V. Wadde11, who also rescued the author from many pitfalls along the way. The author is also grateful to the following people for helpful discussions and comments during the development of this mode1: L. A. Berry, P. J. Catto, J. F. Clarke, T. H. Dupree, K. Molvig, O. P. Manley, M. N. Rosenbluth, P. H. Rutherford, A. Samain, D. Tetrault, S. J. Zweben, and S. Yoshikawa.

As in the development of any new model, this work has a number of historical antecedents. In the interest of indicating how symbiotic such developments are in the international plasma fusion community, a brief historical account of the development of this particular model will be presented. The first direct stimulus for this work came from B. B. Kadomtsev, who, in an informal discussion with the author in Dubna in July 1975, emphasized the role that field errors or magnetic perturbations might have on plasma transport in tokamaks. In discussing this, Kadomtsev referred to a paper he had just recently published ${ }^{21}$ as a "phenomenological" calculation and hoped it would serve as a "stimulus to young theorists" to find a more appropriate magnetic perturbation mechanism and concomitant effects. It might be said that the development 
of this model is a response to this challenge. The next direct stimulus was provided by M. Cotsaftis, who, in a visit to ORNL in September of 1975, presented his work on and emphasized the role that the very anisotropic electron heat conductivity tensor could have on the magnetic perturbations (in this case tearing modes) in a resistive tokamak. An indirect stimulus at about the same time came from the work by Hazeltine and Strauss, ${ }^{4}$ who showed that within the magnetic island produced by a tearing mode, one has $x_{e r} \sim \dot{X}_{e l}+\left(\tilde{B}_{r} / B\right)^{2} x_{e l l}$. Other indiroct $\varepsilon$ timuli came from the heat pulse propagation work by G. L. Jahns and the nuthor, 22 who found that the radial electron heat transport process in a tokamak is a diffusive one, and from the development of the internal disruption mode 1,5 by Waddel1, Jahns, Hicks, and the author, which reemphasized the fact that the magnetic flux surfaces are not rigid concentric tori in a tokamak. At the same time, work with K. T. Tsang and M. Murakami in trying to compare conventional trapped electron mode theory to experimont was producing progressively more discouraging resulls; the theorcticai growth rates and quasilinearly estimated electron heat conduction coefficients seemed to be much smaller than those observed in the experiments. All of thesé stimuli canle to a head at the Borchlesgader IAEA meeting in October 1976. There, B. B. Kadomtsev, in trying to offer a possible explanation for the apparent (but since resolved ${ }^{23}$ ) anomaly between the $x_{e}$ determined by the heat pulse propagation ${ }^{22}$ compared to that of the background plasma, strongly emphasized how fragile the magnetic field lines in tokamaks are to small helical perturbations--a fact that is most clearly seen in the helical magnetic coordinate systems. ${ }^{16}$ Also, in informal discussions, P. Rebut and A. Samain emphatically stressed 
their feelings that somehow magnetic field lines in tokamaks were ergodic in nature, or at least not stationary in time but rather wiggling a bit. In addition, it appeared' from the controversy at the meeting concerning the observed fluctuation levels and induced radial transport in ATC and TFR that a quasilinear type $\underline{\tilde{E}} \times \underline{B}$ effect of the fluctuations might not be sufficient to explain the experimentally observed radial electron heat transport. The hypothesis developed in this paper finally jelled as a result of an informal discussion with J. B. Taylor at the meeting, in which (in addition to emphasizing the possible effects of magnetic inhomogeneities on the shear stabilization of drift waves) he said: "it would seem that the anomalous transport is due to some indirect effect of drift waves on the magnetic configuration." While the author does not recall precisely what Taylor was thinking about in making this remark, it triggered in him the recollection (from some previous work on trapped electron drift instabilities of which he had been a part ${ }^{10}$ ) that all drift waves at any nonzero $\beta$ have a magnetic component to them. $i$ Thus the present model was developed to illuminate indirect effects of drift waves. 


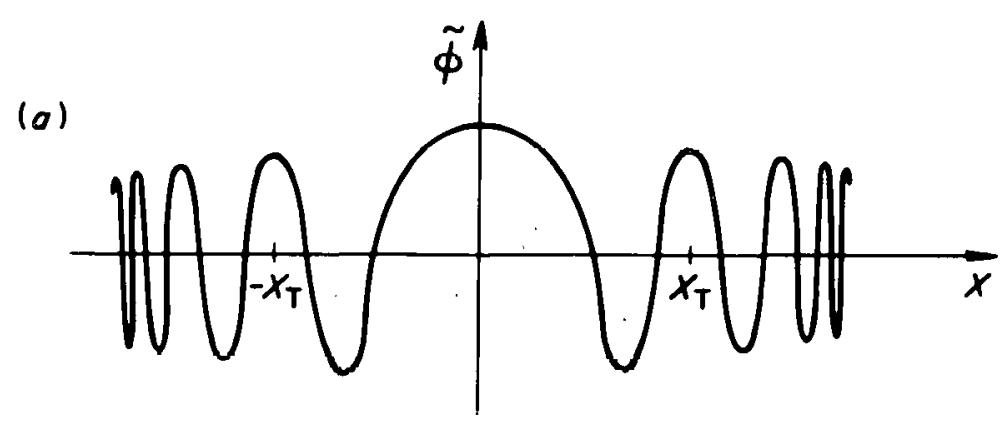

(b)
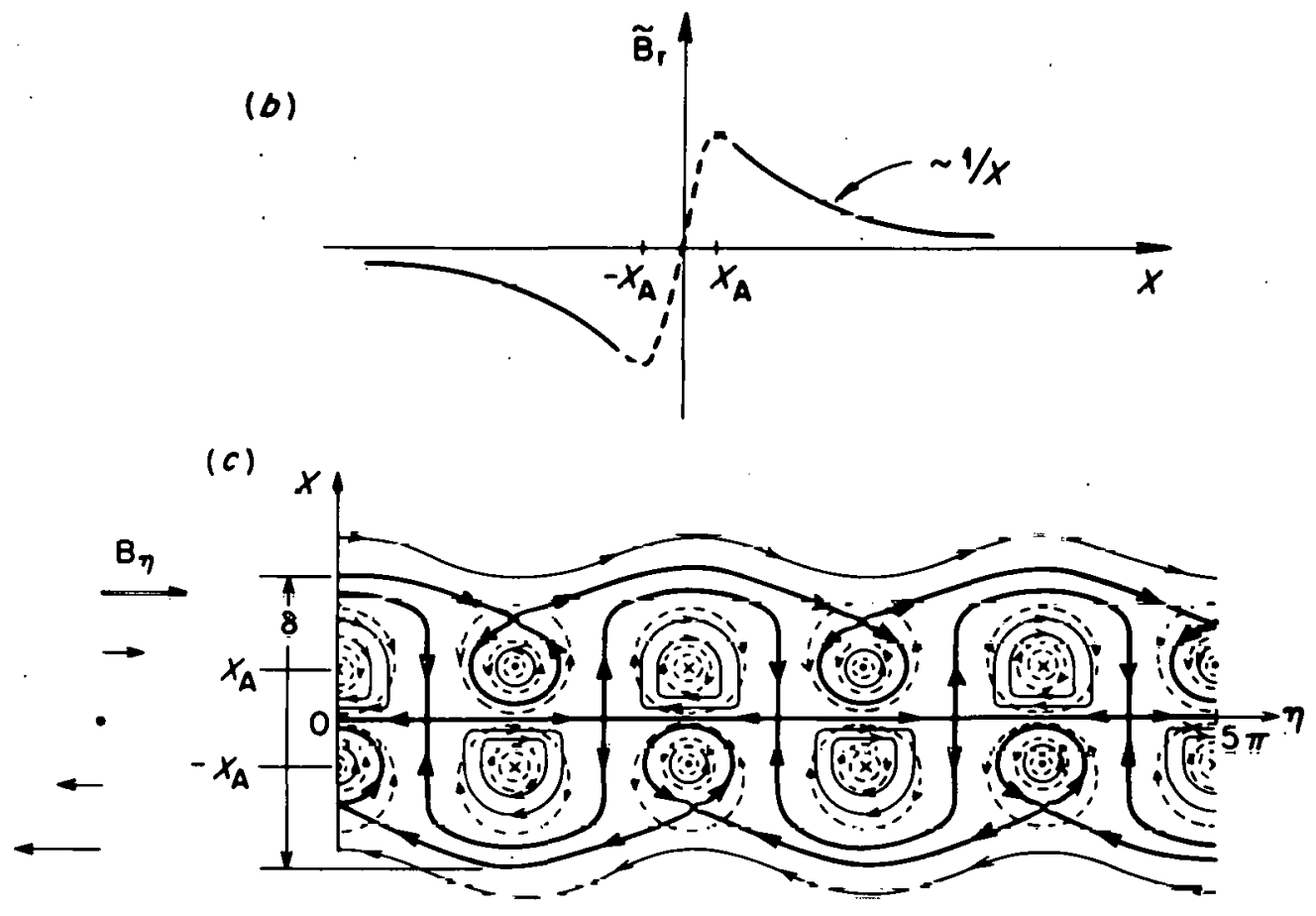

Fig. 1. Schematic illustration of the spatial structure of: (a) the perturbed potential $\tilde{\phi}_{\text {; }}$ (b) the magnetic field perturbation $\tilde{\mathrm{B}}_{\mathrm{r}}$; and (c) the magnetic island structure formed by the combination of $\tilde{B}_{r}$ and the cquilibrium magnetic fields in the vicinity of a rational surface where the helical component of the equilibrium magnetic field is $B_{n} \simeq B_{\theta}^{-} \times q^{*} / q$. The radial distance $x_{A} \equiv\left(L_{s} / r_{n}\right)\left(V_{s} / V_{A}\right) \hat{p}_{i}$ is the spatial point at which the phose velocity of the wave is equal to the Alfven speed; i.e., $\omega / k_{\|}\left(x_{A}\right)=V_{A}$. The distance $x_{T} \sim \sqrt{T_{e} L_{s} / r_{n} T_{i}} \hat{\rho}_{i}$ is the turning point distance of the potential function eigenmode, which is of the form $10 \sim \exp \left(-i x^{2} / x_{T}^{2}\right)$. 


\section{REFERENCES}

1. A. I. Morozov and L. S. Solov'ev, "The Structure of Magnetic Fields,": p. 1 et seq in Reviews of Plasma Physics, Vo1. 2, ed. M. A. Leontovich Consultants Bureau, New York, 1966.

2. S. Matsuda and M. Yoshikawa, Jap. J. App1. Phys. 14, 87 (1975);

P. Chrisman, J. F. Clarke, and J. A. Rome, Magnetic Is land Formation in a Tokamak Plasma From Helical Perturbations of the Plasma Current, ORNL-TM-4501, Oak Ridge National Laboratory, Oak Ridge, Tennessee (March 1974).

3. B. B. Kadomtsev, Fiz. Plazmy $\underline{1}$, 710 (1975) [Sov. J. Plasma Phys. 1 , 389 (1976) ] ; B. V. Wadde11, M. N. Rosenbluth, D. A. Montice11o, and R. B. White, Nucl. Fusion 16, 528 (1976).

4. J. A. Krommes and P. H. Rutherford, Nuc1. Fusion 14, 695 (1974); R. D. Haze1tine and H. R. Strauss, Phys. Rev. Lett. 37, 102 (1976); M. Cotsaftis, Bul1. Am. Phys. Soc. 20, 2160 (1975).

5. B. V. Wadde11, G. L. Jahns, J. D. Callen, and H. R. Hicks, "Interpretation of Tokamak Sawtooth Oscillations" (submitted to Phys. Rev. Lett., February 1977); "Internal Disruptions in Tokamaks," ORNL/TM-5840, Oak Ridge National Laboratory, Oak Ridge, Tennessee (May 1977).

6. B. B. Kadomtsev, "Reconnection of Field Lines and Disruptive Instability in Tokamaks;" paper B1 at 6th Int1. Conf. on Plasma Physics and Controlled Nuclear Fusion Research, Berchtesgaden, FRG, October 6-13, 1976; proceedings to be published.

7. A. Samain, Plasma Phys. 18, 551 (1976); A. B. Rechester and T. H. Stix, Phys. Rev. Lett. 36, 587 (1976).

8. J. L. Dunlap, J. H. Harris, and V. K. Pare, Bul1. Am. Phys. Soc. 21, 1051 (1976). 
9. E. Mazzlicato, Phys, Rev. Lett, 36, 792 (1976); C. M. Surko and R. E. Slusher, Phys. Rev. Lett. 37, 1747 (1976); TFR Group, "An Energy Bàlance Study of a TFR Discharge," paper A3, at 6th Int1. Conf. on Plasma Physics and Controlled Nuclear Fusion Research, Berchtesgaden, FRG, October 6-13, 1976; proceedings to be published.

10. W. M. Tang, C. S. Liu, M. N. Rosenbluth, P. J. Catto, and J. D. Callen, Nuc1. Fusion 16,191 (1976). See also Ref. 14.

11. M. G. Rusbridge, Plasma Fhys. 11,35 (1969). The author is grateful to E. Apgar for pointing out the existence of this work on a similar hypothesis.

12. A. Rechester, "Electron Heat Transport in a Tokamak with Destroyed Magnetic Surfaces," paper E15 at Annual Controlled Fusion Theory Conference, San Diego, California, May 4-6, 1977.

13. B. B. Kadomtsev, pp. 82-83 in Plasma Turbulence, Academic Press, London, 1965.

14. P. J. Catto, A. M. El-Nadi, C. S. Liu, and M. N. Rosenbluth, Nucl. Fusion 14, 405 (1974).

15. J. D. Callen, G. G. Kelley, and B. V. Waddell, "Magnetic Island Characteristics in Tokamaks" (to be published as an ORNL/TM).

16. B. B. Kadomtsev and 0. P. Pogutse, Zh. Eksp. Teor. Fiz. 65, 575 (1973) [Sov. Phys. - JETP $\underline{38}, 283(1974)]$.

17. 'I. H. Dupree, Phys. Fluids 10, 1049 (1967).

18. J. F. Drake and Y. C. Lee, "Kinetic Theory of Tearing Instabilities," Bull. Am. Yhys. Soc. 21,1091 (1976); paper PA5 at Annual Controlled Fusion Theory Conference, San Diego, California, May 4-6, 1977; Liu Chen, P. H. Rutherford, and W. M. Tang, "Drift-Tearing Instabilities Due to Trapped Elestrons," paper PA4 at Annual Controlled Fusion Theory Conference, San Diego, California, May 4-6, 1977. 
19. S. von Goeler, W. Stodiek, N. Sauthoff, and H. Selberg, "X-ray Spectra in the ST Tokamak," paper presented at the 3rd Int1. Symposium on Torodial Plasma Confinement, Garching, FRG, March 26-30, 1973;

S. Sesnic and G. Fussmann, Runaway Bremsstrahlung Spectra in Pulsator Tokamak, Report IPP III/29, Max-Planck Institut für Plasmaphysik, Garching, FRG (August 1976); J. C. Strachan, Nuc1. Fusion 16, 743 (1976).

20. S. J. Zweben, D. W. Swain, J. B. Wilgen, and H. H. Fleischman, Bull. Am. Phys. Soc. $\underline{22}, 182$ (1977).

21. B. B. Kadomtsev, Fiz. Plazmy 1, 938 (1975) [Sov. J. Plasma Phys. 1 , $512(1975)]$.

22. J. D. Callen and G. L. Jahns, Phys. Rev. Lett. $\underline{38}, 491$ (1977).

23. M. Soler (Junta de Energia Nuclear, Madrid, Spain), private communication, April 1976 at ORNL. 


\section{THIS PAGE \\ WAS INTENTIONALLY \\ LEFT BLANK}




\section{INTERNAL DISTRIBUTION}

ORNL/TM-5974.

1. J. D. Callen

2. J. F. Clarke

3. R. A. Dory

4. G. G. Kelley

5. O. B. Morgan

6. M. W. Rosenthal

7-31. J. D. Callen

32-34. Laboratory Records Department

35. Laboratory Records, ORNL - RC

36. Y-12 Document Reference Section

37-38. Central Research Library

39. Fusion Energy Division Library

40. Fusion Energy Division Reports Office

41. ORNL Patent Office

\section{EXTERNAL DISTRIBUTION}

42. Plasma Physics Library, Plasma Physics Laboratory, Princeton Univ., Forrestal Campus, P.0. Box 451, Princeton, NJ 08540

43. Controlled Thermonuclear Research Library, Lawrence Livermore Laboratory, P.0. Box 808, Livermore, CA 94550

44. Q Division Library, Los Alamos Scientific Laboratory, P.0. Box 1663, Los Alamos, NM 87544

45. Controlled Thermonuclear Research Library, c/o Weston M. Stacey, Jr., Argonne National Laboratory, 9700 S. Cass Ave., Argonne, IL 60439

46. CTR Computer Center, c/o Dr. John Killeen, Lawrence Livermore Laboratory, P.0. Box 808, Livermore, CA 94550

47. Librarian, Culham Laboratory, U.K. Atomic Energy Authority, Abingdon, Oxon, OX14. 3DB, United Kingdom

48. Ruth Lengye, Bibliothek, Max-Planck Institut für Plasmaphysik, 8046 Garching bei München, Federal Republic of Germany

49. Library, Centre de Recherches en Physique des Plasmas, 21 Avenue des Bains, 1007, Lausanne, Switzerland

50. A. M. Dupas, Documentation S.I.G.N., Départment de 1a Physique du Plasma et de la Fusion Controlée, Association EURATOM-CEA sur la Fusion, Centre d'Etudes Nucléaires, BP 85 Centre Du TRI 38041 Grenoble Cedex (France)

51. Bibliothèque, Service du Confinement des Plasmas, C.E.A., B.P. No. 6, 92, Fontenay-aux-Roses (Seine) France

52. Library, International Centre for Theoretical Physics, Trieste, Italy

53. Library, Laboratorio Gas Ionizzati, Frascati, Italy

54. V. E. Ivanov, Physical-Technical Institute of the Ukranian Academy of Sciences, Sukhumi, U.S.S.R.

55. L. M. Kovrizhnikh, Lebedev Institute of Physics, Academy of Sciences of the U.S.S.R., Leninsky Prospect 53, Moscow, U.S.S.R.

56. Prof. Dshumber G. Lominadze, Academy of Sciences of the Georgian SSR, 8 Dzerzhinski St., 38004, Tbilisi, USSR

57. Library, Inst. for Plasma Physics, Nagoya Univ., Nagoya, Japan 464 
58. Library, FOM-Institut voor Plasma-Fysica, Rijnhuizen, Jutphaas, Netherlands

59. Plasma Physics Group, Department of Engineering Physics, Australian National University, P.0. Box 4, Canberra A.C.T. 2600, Australia

60. Thermonuclear Library, Japan Atomic Energy Research Institute, Tokai, Naka, Ibaraki, Japan

61. Dr. D. G. McAlees, Exxon Nuclear Co., Inc., Research \& Technology Laser Enrichment Department, 2955 George Washington Way, Richland, WA 99352

62. CTR Reading Room, c/o Prof. D. W. Kerst, Dept. of Physics, Sterling Ha11, Univ. of Wisconsin, Madison, WI 53706

63. CTR Reading Room, c/o Prof. I. B. Bernstein, Yale Univ., 200 Mason Laboratory, Dept. of Engineering \& Applied Science, New Haven, CT 06510

64. Center for Plasma Physics and Thermonuclear Research, ç/o D. W. Rọss, Physics Dept., Univ. of Texas, Austin, TX 78712

65. CTR Reading Room, c/o Prof. B. D. Fried, Physics Dept., Univ. of California, Los Angeles, CA 90024

66. CTR Reading Room, c/o Prof. David C. Montgomery, Physics \& Astronomy Dept., Univ. of Iowa, Iowa City, IA 52240

67. Magneto-Fiuid-Dynamics Library, $c / 0 \mathrm{Dr}$. Harold Grad, Courant Inst. of Math. Sci., New York Univ., 251 Mercer St., New York, NY 10012

68. CTR Reading Room, c/o Prof. Allan N. Kaufman, Physics Dept., Univ. of California, Berkeley, CA 94720

69. Dr. David A. Dingee, Fusion Programs, Battelle-Northwest, Battelle Boulevard, Richland, WA 99352

70. CTR Reading Room, c/o Prof. C. S. Liu, Dept. of Physics and Astronomy, Univ. of Maryland, College Park, MD 20742

71. CTR Reading Room, c/o Prof. T. Kammash, 103 Research Admin. Bldg., N. Campus, Univ. of Michigan, Ann Arbor, MI 48105

72. CTR Reading Room, c/o Dr. Ravi N. Sudan, Phillips Hall, Cornell Univ., Ithaca, NY 14850

73. Prof. Marshal1 N. Rosenbluth, Institute for Advanced Study, Princeton, NJ 08540

74. CTR Reading Room, c/o Prof. R. Gross, Plasma Research Lab., Columbia Univ., New York, NY 10027

75. CTR Reading Room, c/o Prof. Roy Gould, California Inst. of Tech., M.S. 116-81, Pasadena, CA 91125

76. Dr. Nicholas A. Krall, Science Applications, Inc., P.0. Box 2354, 1200 Prospect St., La Jolla, CA 92037

77. CTR Reading Room, c/o Dr. Jay P. Boris, Plasma Physics, Naval Research Laboratory, Washington, DC 20390

78. Professor A. Simon, Dept. of Mechanical \& Aeruspace Sciences, University of Rochester, Rochester, NY 14627

79. CTR Library, c/o Dr. Alan F. Haught, United Technologies Research Labs, East Hartford, CT 06108

80. Dr. H. K. Forsen, Exxon Nuclear Co., Inc. 777-106th Avenue, NE, C-000\%7\%, Bellevue, WA 98009

81. Dr. George Vahala, Physics Dept., College of William \& Mary, Williamsburg, VA 23185

82. Dr. Robert E. Price, Division of Magnetic Fusion Energy, G-234, Energy Research and Development Administration, Washington, D.C. 20545 
83. Dr. R. C. Davidson, Division of Magnetic Fusion Energy, G-234, Energy Research and Development Administration, Washington, D.C. 20545

84. Dr. Oscar P. Manley, Division of Magnetic Fusion Energy, G-234, Energy Research and Development Administration, Washington, D.C. 20545

85. Mr. E. E. Kintner, Division of Magnetic Fusion Energy, G-234, Energy Research and Development Administration, Washington, D.C. 20545

86. Dr. L. D. Pearlstein, L-388, Lawrence Livermore Laboratory, P.0. Box 808, Livermore, CA 94550

87. Dr. J. P. Friedberg, Los Alamos Scientific Laboratory, Los Alamos, NM 87544

88. Dr. David J. Rose, Dept. of Nuclear Engineering, MIT, Cambridge, MA 02139

89. Dr. Gareth E. Guest, General Atomic Co., P.0. Box 81608, San Diego, CA 92138

90. Dr. Claude Mercier, Service du Theorie des Plasmas, Centre d'Etudes Nucléaires, Fontenay-aux-Roses (seine) France

91. Dr. J. B. Taylor, Culham Laboratory, UKAEA, Abingdon, Oxon, OX14 3DB, United Kingdom

92. Dr. D. Pfirsch, Institute for Plasma Physics, 8046 Garching bei München, Federal Republic of Germany

93. Dr. V. D. Shafranov, I. V. Kurchatov Inst. of Atomic Energy, 46 Ulitsa Kurchatova, P.0. Box 3402, Moscow, U.S.S.R.

94. Dr. A. Rogister, Institute for Plasma Physics, KFA, Postfach 1913, D-5170, Jülich 1, Federal Republic of Germany

95. Dr. J. G. Cordey, Culham Laboratory, UKAEA, Abingdon, Oxon, OX14 3DB, United Kingdom

96. Dr. David Baldwin, L-388, Lawrence Livermore Lab., P.0. Box 808, Livermore, CA 94550

97. CTR Reading Room, c/o Prof. Bruno Coppi, Dept. of Physics, MIT, Cambridge, MA 02138

98. Dr. Harold P. Furth, Princeton Plasma Physics Lab., Princeton Univ., P.0. Box 451, Princeton, NJ 08540

99. Dr. Paul H. Rutherford, Princeton Plasma Physics Lab., Princeton Iniv, P.O. Box 451, Princeton, NJ 08540

100. Research \& Technical Support Div., Oak Ridge Operations, Energy Research and Development Administration, P.O. Box E, Oak Ridge, TN 37830

101-127. Tech. Information Center, P.0. Box 62, Oak Ridge, TN 37830 